\title{
UMA PROPOSIÇÃO METODOLÓGICA PARA A AVALIAÇÃO DA COMPACTAÇÃO DE PÓS
}

\author{
Professor Mestre Alexandre Alvarenga Palmeira (Engenharia Mecânica - UniFOA) - \\ alexandre.palmeira@foa.org.br
}

Professor Mestre Alexandre Fernandes Habibe (Engenharia Mecânica - UniFOA) alexandre.habibe@foa.org.br

Professor Doutor José Glênio Medeiros de Barros (Engenharia de Produção - UERJ) glenio@uerj.br

Professor Doutor Cyro Alves Borges Júnior (Engenharia de Mecânica - UERJ) cyroborges@globo.com

\section{Resumo}

O processo de produção de um produto a partir de pós, em geral, segue as seguintes etapas: Obtenção dos Pós, Preparação e Mistura dos Pós, Conformação dos Pós, Sinterização e Acabamento. Dentre os processos de conformação dos pós está a prensagem a seco, nele a peça obtida apresenta uma distribuição de densidade não uniforme no seu interior. Tal variação de densidade de compactação é função de vários fatores, como: uso de lubrificantes nas paredes do molde ou na mistura, morfologia do pó, tamanho médio das partículas, distribuição de tamanho de partículas, de direção de aplicação da pressão, ou seja, se compactado em prensas de ação simples ou dupla ação, etc.. Este trabalho objetiva apresentar uma nova proposta de metodologia de avaliação da compactação de pós pelo método de elementos finitos. Com essa metodologia leva-se em consideração a forma, tamanho e distribuição dos pós no interior do molde, tornando o modelo mais realístico. Pois leva em consideração fatores importantes, como: a morfologia do pó, tamanho médio das partículas, distribuição de tamanho de partículas, etc.. Sendo assim, espera-se poder avaliar e estimar vários fatores e parâmetros de operação, como a curva ideal de aplicação de pressão, obter através da simulação a comprovação numérica do Coeficiente de Andreasem. Obter uma distribuição morfológica de pós que produza a distribuição de densidade mais homogênea, avaliar qual o tamanho médio e distribuição de partículas que também produzam uma distribuição densidade mais homogênea. 
Palavras-chave: Compactação de pós, Metodologia, Elementos Finitos

\section{Introdução}

A obtenção de produtos rígidos a partir de pós, cerâmicos ou metálicos, foi uma das primeiras tecnologias dominadas pelo homem. Atualmente a obtenção de produtos através da tecnologia do pó tem sido amplamente utilizada, não só para obtenção de produtos a partir das chamadas cerâmicas naturais, mas também para obtenção de produtos para indústria automotiva e de eletrodomésticos, entre outras, revelando-se como a melhor solução na produção de peças em larga escala, a um baixo custo, com uma grande precisão dimensional.

O processo de produção de um produto a partir de pós, em geral, segue as seguintes etapas: $1^{\circ}$ Obtenção dos Pós, $2^{\circ}$ Preparação e Mistura dos Pós, $3^{\circ}$ Conformação dos Pós, $4^{\circ}$ Sinterização e $5^{\circ}$ Acabamento. Uma etapa de vital importância é a conformação dos pós que, entre outros, pode ser realizada por prensagem a seco.

\section{Prensagem a Seco de Pós}

Segundo Norton (1973), esse processo é utilizado na fabricação de pequenos isoladores elétricos, ladrilhos, azulejos, telhas e refratários. O processo, também, é utilizado na produção de velas de ignição de veículos automotivos, é caracterizado pela grande uniformidade e alta qualidade dos produtos obtidos. Tornando o processo cada vez mais popular na indústria para fabricação de componentes cerâmicos de precisão.

Porém um dos problemas da prensagem a seco, segundo Norton(1973), é obtenção de uma densidade não uniforme no interior da peça compactada. Tal variação de densidade de compactação é função de vários fatores, como: uso de lubrificantes nas paredes do molde ou na mistura, morfologia do pó, tamanho médio das partículas, distribuição de tamanho de partículas, de direção de aplicação da pressão, ou seja, se compactado em prensas de ação simples ou dupla ação, etc..

\section{Modelagem Do Pó Cerâmico}

De acordo com Chiaveini (2001), ao se considerar os dois processos mais comuns de compactação, compactação em matriz rígida e compactação isostática em invólucros ou receptáculos flexíveis, o comportamento do pó pode-se dividir em três estágios:

$1^{\circ}$ ) As partículas do pó são re-amontoadas e ou re-arranjadas;

$2^{\circ}$ ) Ocorre deformação plástica das partículas; 
$\left.3^{\circ}\right)$ As partículas do pó fícam frágeis devido ao encruamento nos estágios anteriores, quebrando e formando fragmentos menores.

Vários autores têm procurado estabelecer fórmulas matemáticas que relacionem a pressão de compactação com a densidade final do material ou sua prosidade.

\section{Comportamento plástico do Pó}

De acordo com Canto (2002), o modelo modificado de Druker-Prager/cap, que foi desenvolvido para representar o comportamento de solos, pode ser utilizado para representar o comportamento plástico de materiais em pó. Esse modelo utiliza a superfície de Drucker-Prager limitada por uma superfície cap no eixo das tensões hidrostáticas.

Segundo Ruddy et al. (2005), o critério de Druker-Prager/cap foi originalmente desenvolvido para predizer a deformação plástica de solos sob ação de forças compressivas. Esse critério consiste principalmente pela interseção de duas superfícies de tensão: a superfície da tensão de cisalhamento $\left(F_{s}\right)$ e a superfície da tensão $\operatorname{cap}\left(F_{c}\right)$, uma superfície de transição $\left(F_{t}\right)$ foi introduzida para promover um perfeito ajuste entre as supercífies. Para um estado uniaxial de compactação as superfícies são definidas no plano p-q, ou seja, a tensão equivalente de $\operatorname{Von} \operatorname{Mises}(q)$ e a tensão média $(p)$, que valem respectivamente:

$$
\begin{aligned}
& p=\frac{1}{3}\left(\sigma_{1}+\sigma_{2}+\sigma_{3}\right) \\
& q^{2}=\frac{1}{6}\left[\left(\sigma_{1}-\sigma_{2}\right)^{2}+\left(\sigma_{2}-\sigma_{3}\right)^{2}+\left(\sigma_{3}-\sigma_{1}\right)^{2}\right]
\end{aligned}
$$

A representação gráfica do critério de escoamento de Druker-Prager/cap é mostrado na Figura 1, a seguir.

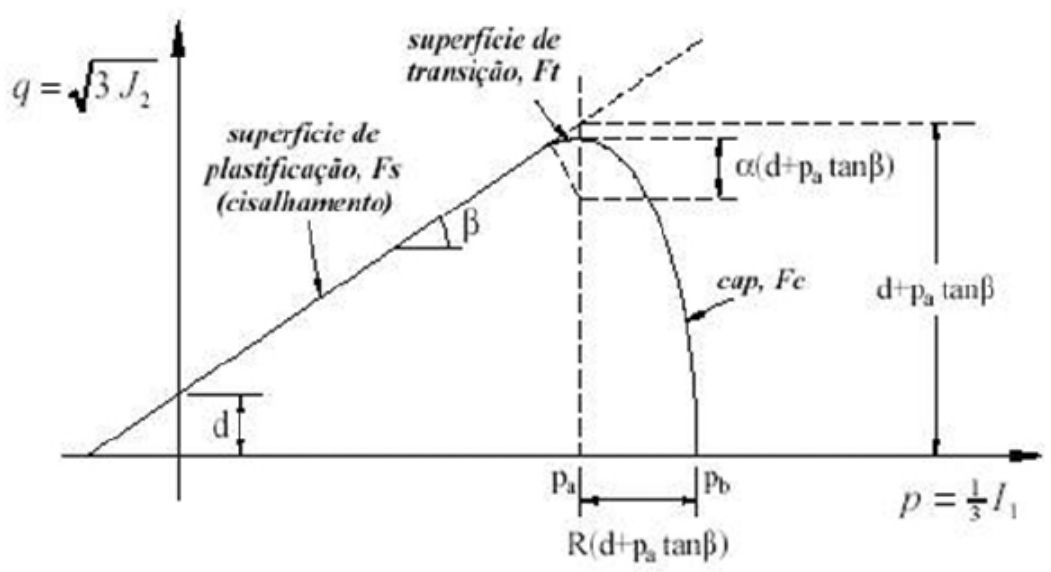

Figura 1: Modelo Druker-Prager/cap nos planos $p$-q. 
Onde $d$ é a coesão, $\beta$ é o ângulo de atrito entre as partículas do material, $R$ é o parâmetro de forma da superfície cap e $a$ o parâmetro de ajuste entre as superfícies.

\section{Densificação do Pó}

Vários autores têm correlacionado a evolução da tensão que atua sobre o pó durante a compactação com a evolução da densidade. De acordo com Ruddy et al. (2005), a densidade pode ser prevista de dois modos:

$1^{\circ}$ ) Ela pode ser calculada pelo volume final do compactado, que é função do deslocamento total do punção.

$2^{\circ}$ ) A densidade média relativa pode ser calculada pela densidade de cada elemento no pó compactado.

Porém, segundo Khoi et al (2003), para se realizar uma análise quasi-nãolinear resultados experimentais devem ser incorporados a simulação numérica da compactação dos pós. Ele estabelece assim uma variação do módulo de Young do material $(E)$ em função da variação da densidade relativa (r), sendo obtido pela equação a seguir:

$$
E=K \rho^{n}
$$

Onde $K$ e $n$ seriam obtidos experimentalmente para cada pó a ser compactado.

Onde $K$ e $n$ seriam obtidos experimentalmente para cada pó a ser compactado.

\section{Método de Elementos Finitos}

A necessidade de redução do tempo e dos custos de geração de um projeto leva a indústria a utilizar métodos de simulação numérica para o desenvolvimento de seus produtos. A equação de governo que rege o equilíbrio em uma análise linear dinâmica no sistema de elementos finitos, é dada por:

$$
M \dot{U}+C \dot{U}+K U=R
$$

onde $M, C$ e $K$ são as matrizes de massa, amortecimento e rigidez respectivamente, e $\ddot{U}$, $U$ e $U$ correspondem aos vetores de aceleração, velocidade e deslocamentos respectivamente e $R$ o vetor de carregamento externo. De acordo com Westheiner et al, citado por Menezes (1995), as técnicas numéricas de integração são classificadas em método de integração implícito e explícito no tempo, devido às equações de governo serem integradas, ambas, no espaço e no tempo.

\section{Método Explícito de Integração no Tempo}

De acordo com Schweizerhof et al (1991) a integração explícita no tempo é para problemas específicos, pois é mais eficiente uma vez que integra a variável tempo em conjunto com o espaço discretizado por elementos finitos em problemas de mecanismos 
estruturais que dependem do tempo. É interessante notar que esse método de integração não necessita de uma fatorização efetiva da matriz de rigidez a cada passo, fato esse que é a chave de sua eficiência computacional, a matriz de massa $M$ é dita concentrada ("lumped mass").

$\mathrm{O}$ uso do procedimento explícito requer um tempo de incremento muito pequeno, pois o método de integração faz necessário o uso de um "time step"- $\Delta t$ que deve ser menor que um valor crítico - $\Delta t_{c r}$ que pode ser calculado em função das propriedades características do sistema (rigidez, amortecimento, massa específica, etc.). Este parâmetro limite de estabilidade, $\Delta t_{c r}$ é dado em termos dos autovalores do sistema, isto é:

$$
\Delta t_{c r} \leq \frac{2}{w_{\max }}
$$

Segundo Rebelo et al (1992), o limite de estabilidade pode ser aproximado pelo menor tempo necessário para que uma onda elástica atravesse qualquer elemento da malha. Em uma análise que dure $T$ segundos, o programa deve executar $N$ incrementos com duração de $\Delta t$ segundos cada um, ou seja, o número total de incrementos, $N$, requeridos para completar a análise é dado por $T / \Delta t$. De acordo com Rebelo et al (1992), no procedimento explícito, o incremento de tempo estável $\left(\Delta t_{c r}\right)$ é tipicamente muito pequeno se comparado com o tempo natural de duração do processo de conformação. Qualquer abordagem para redução do tempo de processamento requerido para analisar uma dada malha precisará aumentar o incremento de tempo $\Delta t$ ou reduzir o tempo total $T$, ou seja, a velocidade do ferramental pode ser aumentada, através do aumento da velocidade do punção (load factoring).

\section{LOAD FACTORING}

Consiste em se reduzir o tempo total de análise $T$, simplesmente diminuindo o tempo da aplicação de cargas e das condições de contorno. A vantagem deste método, é que as propriedades do material permanecem inalteradas, contudo essa abordagem, pode também fornece resultados errados se a velocidade do processo for aumentada além de certos limites. Com o aumento da velocidade dos componentes a energia cinética também aumenta e pode eventualmente atingir a mesma ordem de grandeza da energia de deformação ou do trabalho feito no material. Não é fácil prever em que nível esse efeito é significativo, mas uma regra baseada na experiência é limitar a energia cinética em no máximo 5\% da energia de deformação.

\section{Método Implícito de Integração no Tempo}

Este método é assim classificado por que a solução da equação de governo que rege o equilíbrio é obtida pela consideração no tempo $t+\Delta t$. Sendo conhecidos o deslocamento e 
a velocidade no tempo $t$, para obter estes valores no tempo $t+\Delta t$, o equilíbrio de forças no tempo é dado pela equação (6):

$$
M \ddot{U}_{t+\Delta t}+C \dot{U}_{t+\Delta t}+K U_{t+\Delta t}=R_{t+\Delta}
$$

O procedimento empregado para solucionar a equação (6) é o método de Newmark de integração no tempo, que utiliza uma expansão em diferenças finitas no intervalo de tempo

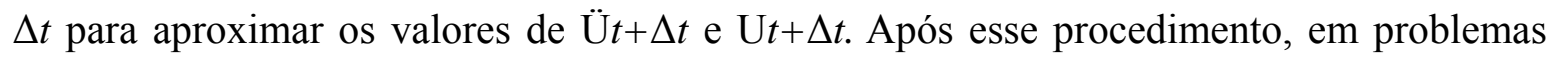
que envolvem não-linearidades, geralmente é utilizado o método incremental de NewtonRaphson para se obter o valor de Üt+ $\Delta t$. Nele o carregamento é prescrito, por exemplo o deslocamento do punção é dividido em pequenos incrementos e em cada um destes é calculado o vetor deslocamento. Esse método requer a montagem e a inversão da matriz de rigidez a cada novo incremento, o que representa um aumento no tempo de processamento e uma maior requisição de memória na análise de modelos tridimensionais.

\section{Elementos e sua Disposição no Modelo}

Um fator que pode tornar onerosa a análise é o número de elementos da malha, pois o tempo de processamento é proporcional ao número de elementos presentes no modelo. Burford et al (1991) observou que os resultados simulados, tanto de medidas de deformação quanto de força, são bastante sensíveis não só ao refinamento da malha mas a disposição dos elementos na mesma.

\section{Formulação do Contato}

Nas análises de conformação é muito importante e complexa a modelagem do contato entre a ferramenta e a peça que está sendo trabalhada, pois se considera possível a interferência, o deslizamento e a perda do contato das mesmas durante a simulação. Na modelagem matemática, adotada no ABAQUS, dependendo da natureza do problema de contato, dois procedimentos podem ser aplicados:

\section{Superfícies de Contato}

Para a estratégia de contato, a superfície cujos nós são testados contra a penetração é chamada de superfície "master" e a do nó penetrante é chamada de "slave". Nesse modelo, cada potencial de contato tem suas condições definidas em termos de duas superfícies em contato. Os nós da superfície slave são restringidos para não penetrar na superfície master. Conseqüentemente a direção de contato é sempre normal à superfície master. Os nós da superfície master podem, em princípio, penetrar na superfície slave. 
Nessa formulação, todas as partes que compõem o ferramental fazem uso de uma formulação de corpo rígido (master) que possui na sua constituição elementos que não se deformam quando entram em contato com outras superfícies (slave). O código de elementos finitos ABAQUS faz uso de dois tipos de formulação de superfície de contato, e são elas: RIGID BODY (Corpo Rígido) e RIGID SURFACE (Superfície Rígida).

\section{a) RIGID BODY - Corpo Rígido}

Esse tipo de formulação é aplicado, em geral, em geometrias tridimensionais. Essa técnica consiste em se criar a geometria do ferramental (master) e utilizar elementos de formulação rígida para compor a mesma. Cada superfície gerada possui um nó de referência onde são aplicas as restrições cinemáticas de movimento do modelo. No nó de referência também são aplicados carregamentos concentrados. Nos demais nós do modelo podem ser aplicados carregamentos distribuídos, como pressão e forças de corpo. Apesar dessa formulação ser utilizada no método implícito e explícito de integração no tempo, ela apresenta diferenças. No método implícito de integração no tempo, o código ABAQUS aplica as restrições de modo que os nós da superfície slave não penetrem na superfície master, mas em princípio, os nós da superfície master podem penetrar na superfície slave. Já quando o código utiliza o método explícito de integração no tempo, a formulação master-slave é "estimada". Assim, as restrições de contato são aplicadas de modo que não ocorra penetração em ambas as superfícies, ou seja, o contato é forçado de modo que as duas superfícies façam o papel de superfície master e slave ao mesmo tempo. Impedindo assim que os nós da superfície slave penetrem nos segmentos da superfície master e viceversa. As restrições de contato são, desta forma, uma média das duas restrições masterslave.

b) RIGID SURFACE - Superfície Rígida

Essa formulação é utilizada para definir superfícies geométricas simples, pela descrição de sua seção transversal com segmentos de linhas e retas. Nesse caso é definido o contorno da superfície a ser gerada e o código gera a superfície com seus respectivos elementos através de critérios internos de escolha, sem que se possa alterar o tipo ou o número de elementos que compõem a superfície. Essa formulação é computacionalmente menos custosa que a formulação anterior. Assim a superfície master gerada possui um nó de referência no qual são aplicados os carregamentos e as restrições cinemáticas do modelo. Essa formulação, no código ABAQUS, só pode ser utilizada em conjunto com o método implícito de integração no tempo. 


\section{Metodologia de Avaliação de Compactação - Atual}

Vários autores como Canto (2002), Ruddy et al. (2005), Khoi et al. (2002 e 2003), Piccolraz et al. (2002), Eriksson et al. (2005), etc., seguem a metodologia exposta pelo fluxograma, a seguir, da Figura 2.

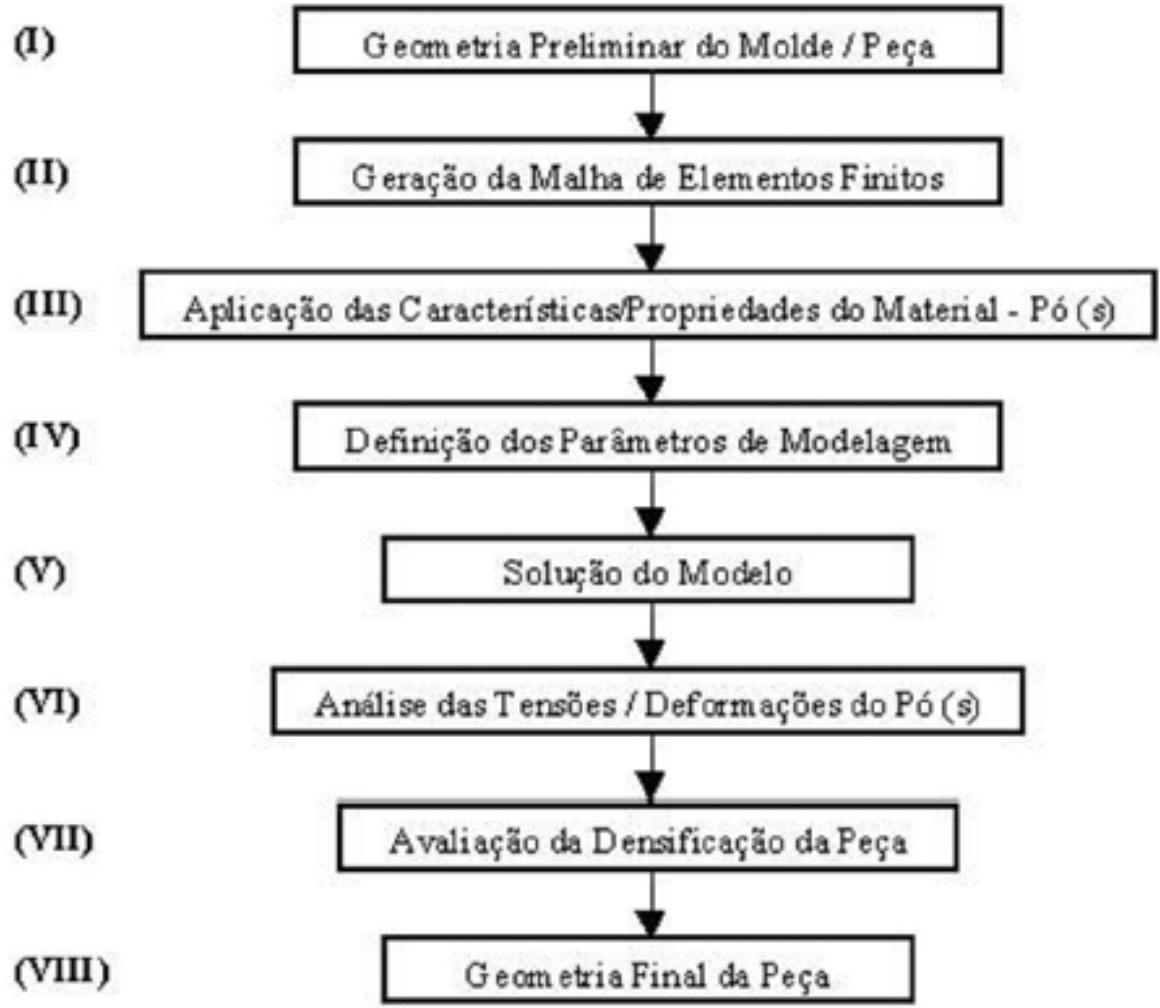

Figura 2: Metodologia de simulação pelo MEF da compactação de pós.

Cabe ressaltar que na etapa (II), a malha de elementos finitos é gerada diretamente em toda a peça, desconsiderando a existência dos vazios naturais gerados pelos poros entre as partículas, ou seja, por esta metodologia o material é considerado como contínuo. $\mathrm{Na}$ etapa (VII) a densificação da peça é obtida de forma indireta, ou pelo estado de tensão da peça, ou pelo estado de deformação da peça, ou pelo deslocamento do punção. Tornando o modelo pouco realístico fisicamente.

\section{Metodologia de Avaliação de Compactação - Nova Proposta}

Este trabalho objetiva apresentar uma nova proposta de metodologia de avaliação da compactação de pós pelo método de elementos finitos. Metodologia exposta pelo fluxograma da Figura 3, a seguir. 
(I)

(II)

Geração das Partículas no Interior do Molde

(III)

(IV)

(v)

(VI)

(VII)

(VIII)

Geração da Malha de Elementos Finitos nas Partículas

Geom etria Preliminar do Molde / Peça
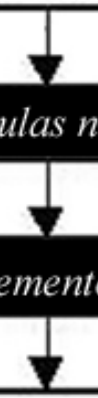

Aplicação das Características/Propriedades do Material - Pó (s)

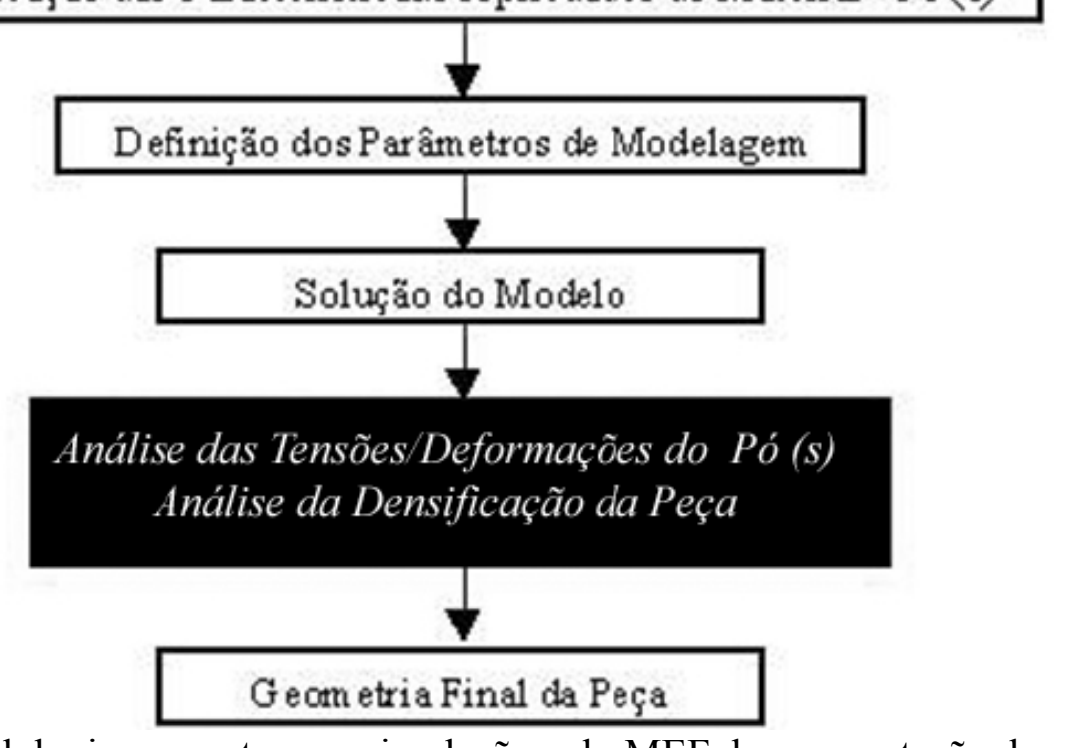

Figura 3: Metodologia proposta para simulação pelo MEF da compactação de pós.

Nesta nova metodologia proposta, na etapa (II) são gerados no interior do Molde as partículas de pós que serão compactadas, levando em consideração sua forma, tamanho e distribuição. Na etapa (III), a malha de elementos finitos é gerada em cada partícula de pó individualmente, considerando assim existência dos vazios naturais gerados pelos poros entre as partículas. Logo etapa (VII) a densificação da peça é obtida de forma direta tornando o modelo mais realístico fisicamente.

\section{Conclusões}

O modelo tradicionalmente utilizado para simulação pelo método de elementos finitos, apesar de apresentar uma boa aproximação com os dados experimentais, segundo a literatura, é pouco realístico e não leva em consideração fatores importantes, como: a morfologia do pó, tamanho médio das partículas, distribuição de tamanho de partículas, etc.. Pois o mesmo trabalha o material no interior do molde, o pó, como sendo contínuo, além disso mesmo obtêm o valor da densificação de forma indireta.

Com esse modelo sugerido para simulação pelo método de elementos finitos, ao se gerar no interior do Molde as partículas de pós que serão compactadas, pode-se levar em 
consideração sua forma, tamanho e distribuição, tornando o modelo mais realístico. Pois se pode levar em consideração fatores importantes, como: a morfologia do pó, tamanho médio das partículas, distribuição de tamanho de partículas, etc..

Sendo assim, espera-se poder avaliar e estimar vários fatores e parâmetros de operação, como a curva ideal de aplicação de pressão, obter através da simulação a comprovação numérica do Coeficiente de Andreasem. Obter uma distribuição morfológica de pós que produza a distribuição de densidade mais homogênea, avaliar qual o tamanho médio e distribuição de partículas que também produzam uma distribuição densidade mais homogênea.

\section{Referências}

- ABAQUS - Theory Manual, Version 5.4 - Hibbitt, Karlsson \& Sorenson Inc., item 2.4.

- ABAQUS User's Manual - Volume I, Version 5.4 - Hibbitt, Karlsson \& Sorenson Inc., item 5.2.1, pp 5.2.1-1 - 5.2.1-5.

- ABAQUS User's Manual - Volume I, Version 5.4 - Hibbitt, Karlsson \& Sorenson Inc., item 8.7.3, pp 8.7.3-1 - 8.7.3-10.

- ABAQUS User's Manual - Volume II, Version 5.4 - Hibbitt, Karlsson \& Sorenson Inc., item 8.4.15, pp 8.4.15-1 - 8.4.15-2.

· ANSYS User's Manual Revision 5.0 - “Theory Manual - Vol. IV”, ANSYS Inc., pp. 17-1 - 17-48, December 1992.

- BURFORD, D. A., NARASIMHAN, K.; WAGONER, R. H. - "A Theoretical Sensitivity Analysis for Full-Dome Formability Tests: Parameter Study for n, m ,r, and m", A Metallurgical Transactions, Vol. 22A, pp 1775-1788, august - 1991.

- CANTO, R. B. "Projeto e Fabricação de Moldes para Prensagem Isostática Utilizando Tecnologias CAD/CAE e Prototipagem Rápida", Tese de Mestrado - Escola de Engenharia de São Carlos-USP, São Carlos, 2002.

- CHIAVERINI, V. - Metalúrgia do Pó, $4^{a}$ Edição. Associação Brasileira de Metalurgia e Materiais, São Paulo, 2001. pp 53.

- COOK, R. D. - Concepts and Applicatiuons of Finite Element Analysis, Edited by John Willey e Sons, Third Edition, pp 376 - 429, New York 1989.

- ERIKSSON, M.; HÄGGBLAD, H.-Å.; HOCKAUF, M.; BERGGREN, C. - "Simulation of High Velocity Compaction of Powder in a Rubber Mould With Characterization of Silicone Rubber and Titanium Powder Using a Modified Split Hopkinson Setup". Powder Tecnology 154, 2005. pp. 33-42.

- KARL SCHWEIZERHOF, K.; HALLQUIST, J. O. - "Explicit Time Integration and Contact Simulations for Thin Sheet Metalforming", International Conference FE- 
Simulation of 3D Sheet Metal Forming Processes in Automotive Industry, Zurich, May 1991.

KHOI, A. R. - "PCS_SUT: a Finite Element Software for Simualation of Powder Forming Processes”. Journal of Materials Processing Technology 125-126, 2002. pp. 602-607.

- KHOI, A. R.; IRANFAR, S. - "3D Numerical Simulation of Elasto-plastic Behaviour in Powder Compaction Porcess Using a Quasi-nonlinear Technique". Journal of Materials Processing Technology 143-144, 2003. pp. 886-890.

- MENEZES, M. A. - "Strain Limit Theories, Anisotropy in Sheet Metal Forming and Simulation of Pressing Processes", Tese de Doutorado - University of Birminghan, Agust 1995.

- NORTON, F. M. - "Introdução a Tecnologia Cerâmica: tradutor, Jefferson Vieira de Souza. Ed. Edgard Blücher, São Paulo, 1973. pp. 131 - 134.

- PICCOLROAZ, A.; GAJO, A.; BIGONI, D. - "Cap5: Forming of Advanced Ceramics”. Selected Mechanical Problems in Structural Ceramics, Polish Academy of Sciencs, Warsaw, Poland, 2002.

- PRIOR, A. M. - "Applications of Implicit and Explicit Finite Element Techniques to Metal Froming”, Journal of Materials Processing Technology - Elsevier Science B.V., pp 649 - 656, 1994.

- REBELO, N.; NAGTEGAAL, J. C.; TAYLOR, L. M. - "Comparasion of Implicit and Explicit Finite Element Methods in the Simulation of Metal Forming Processes", Numerical Methods in Industrial Forming Process, pp 99 - 108, Rotterdam, 1992.

- RUDDY, O. M.; WU, C.-Y.; BENTHAM, A. C.; HANCOCK, B. C.; BEST, S. M.; ELLIOT, J. A. - "Modelling the Mechanical Behaviour of Pharmaceutical Powder During Compaction”. Powder Tecnology 152, 2005. pp. 107-117.

\section{Informações bibliográficas:}

Conforme a NBR 6023:2002 da Associação Brasileira de Normas Técnicas (ABNT), este texto científico publicado em periódico eletrônico deve ser citado da seguinte forma:

PALMEIRA, A. A.; HABIBE, A. F.; BARROS, J. G. M.; ALVES BORGES, C., Jr.. Uma Proposição Metodológica Para A Avaliação Da Compactação De Pós. Cadernos UniFOA, Volta Redonda, ano 2, n. 3, mar. 2007. Disponível em:

$<$ http://www.unifoa.edu.br/pesquisa/caderno/edicao/03/34.pdf $>$ 